\title{
Sacubitril/valsartan: a cardiovascular drug with pluripotential actions
}

\author{
Steven G. Chrysant ${ }^{1}$, George S. Chrysant ${ }^{2}$ \\ ${ }^{1}$ University of Oklahoma Health Sciences Center, Oklahoma City, OK, USA; ${ }^{2}$ INTEGRIS Baptist Medical Center, Oklahoma City, OK, USA \\ Correspondence to: Steven G. Chrysant, MD, PhD. University of Oklahoma Health Sciences Center, 5700 Mistletoe Court, Oklahoma City, OK \\ 73142, USA. Email: schrysant@yahoo.com. \\ Provenance: This is an invited Editorial commissioned by the Section Editor Yue Liu (The First Affiliated Hospital, Harbin Medical University, \\ Harbin, China). \\ Comment on: Engeli S, Stinkens R, Heise T, et al. Effects of sacubitril/valsartan on exercise-induced lipid metabolism in patients with obesity and \\ hypertension. Hypertension 2018;71:70-7.
}

Submitted May 03, 2018. Accepted for publication May 30, 2018.

doi: $10.21037 /$ cdt.2018.05.10

View this article at: http://dx.doi.org/10.21037/cdt.2018.05.10

\section{Introduction}

Sacubitril/valsartan is a first-in-class dual action molecule of the neprilysin (NEP) inhibitor sacubitril (AHU-377) and the angiotensin II (Ang II) type 1 (AT1) receptor blocker (ARB) valsartan. The drug has been approved by both the Food and Drug Administration (FDA) and the European Medicines Agency (EMA) in 2015 for the treatment of heart failure with reduced ejection fraction [HF reduced $\mathrm{EF}(\mathrm{HFrEF})]$ and awaits approval for the treatment of hypertension. Several recent studies have shown it to be effective in reducing the symptoms of HFrEF and in lowering the blood pressure (BP) compared to monotherapy and it is fairly safe and well tolerated (1-7). The beneficial antihypertensive and HF effects of sacubitril/valsartan are mediated through the inhibition of NEP in catabolizing the natriuretic peptides (NPs) and the blockade of Ang II, AT1 receptor with valsartan. These actions of sacubitril/ valsartan result in systemic vasodilation and increased diuresis and natriuresis, leading to decrease in peripheral vascular resistance and plasma volume contraction, all important actions for the lowering of $\mathrm{BP}$ and improving $\mathrm{HF}$ symptoms $(8,9)$. The NPs, atrial NP (ANP) and brain NP (BNP) exert their effects through the generation of cyclic guanosine monophosphate (GNP), but their half-lives are very short being catabolized by various enzymes including NEP. Therefore, one of the beneficial effects of sacubitril/ valsartan is due to the inhibition of NP catabolism and prolongation of their half-lives (9). In addition, recent studies indicate that the NPs are involved in the metabolic homeostasis by targeting multiple organs such as brain, liver, brown fat, skeletal muscle and pancreas, leading to weight loss and improving the glucose metabolism. However, their levels are reduced in obese individuals leading to the development of the metabolic syndrome, type 2 diabetes mellitus and hypertension $(10,11)$. In a recent study, Engeli et al. (12), investigated another possible beneficial effect of sacubitril/valsartan in combination with exercise in obese hypertensive patients through reduced body fat content by lipolysis, increased insulin sensitivity, energy expenditure and inhibition of NP catabolism. The present editorial will be based on the findings of this study in addition to other actions of sacubitril/valsartan.

\section{Antihypertensive effects of sacubitril/valsartan}

The antihypertensive effects of sacubitril/valsartan have been demonstrated by several randomized clinical trials (RCTs) and the results of the main ones will be discussed here, whereas the results of the minor ones are summarized in Table 1. Ruilope et al. (3) were the first ones to report on the BP lowering effects of sacubitril/valsartan (LCZ-696) in single daily doses of 100,200 , and $400 \mathrm{mg}$ versus single daily doses of valsartan 80,160 , and $320 \mathrm{mg}$, versus placebo in 1,216 patients mean age 53 years, with uncomplicated hypertension. The patients were followed for 8 weeks and their BP was measured by clinic as well as ambulatory BP measurement (ABPM). Sacubitril/valsartan dose- 
Table 1 Antihypertensive effects of sacubitril/valsartan alone or versus a comparator

\begin{tabular}{|c|c|c|c|c|c|c|c|}
\hline Author & Pts $(n)$ & Age (years) & F-U (weeks) & $\mathrm{SBP}(\mathrm{mmHg})$ & $\Delta(\mathrm{mmHg})$ & $\mathrm{DBP}(\mathrm{mmHg})$ & $\Delta(\mathrm{mmHg})$ \\
\hline \multicolumn{8}{|l|}{ Kario (6) } \\
\hline LCZ696 200 & 35 & 51 & 4 & 173 & -23.1 & 112 & -14 \\
\hline LCZ696 400 & 32 & 51 & 8 & 173 & -35.3 & 112 & -22.1 \\
\hline \multicolumn{8}{|l|}{ Ito (13) } \\
\hline LCZ696 100 & 32 & 66 & 4 & 152 & -13.4 & 87 & -5.2 \\
\hline LCZ696 200 & 26 & 66 & 6 & 152 & -19.4 & 87 & -7.8 \\
\hline LCZ696 400 & 18 & 66 & 8 & 152 & -20.7 & 87 & -8.4 \\
\hline \multicolumn{8}{|l|}{ Wang (14) } \\
\hline LCZ696 200+ Aml 5 & 123 & 55 & 8 & 138 & -13.9 & 86 & -0.8 \\
\hline Aml 5 & 128 & 55 & 8 & 140 & -8.0 & 86 & -0.3 \\
\hline
\end{tabular}

$\Delta$, change from baseline; Pts, patients; F-U, follow-up; Aml, amlodipine.

dependently, reduced the BP from baseline by $3.2 / 4.7 \mathrm{mmHg}$ with the $100 \mathrm{mg}$ dose, by $11.0 / 6.1 \mathrm{mmHg}$ with the $200 \mathrm{mg}$ dose and by $12.5 / 6.9 \mathrm{mmHg}$ with the $400 \mathrm{mg}$ dose compared to placebo. Similarly, valsartan dose-dependently reduced the BP from baseline by $4.7 / 2.4 \mathrm{mmHg}$ with the $80 \mathrm{mg}$ dose, by $5.7 / 3.2 \mathrm{mmHg}$ with the $160 \mathrm{mg}$ dose and by $12.5 / 4.2 \mathrm{mmHg}$ with the $320 \mathrm{mg}$ dose compared to placebo. In this study, both drug regimens produced similar BP reductions. Similar results were obtained with the ABPM measurements. Kario et al. (5) studied the effects of sacubitril/valsartan in 389 Asian patients with uncomplicated hypertension mean age 52 years, treated with daily doses of 100, 200, and $400 \mathrm{mg}$ compared to placebo and were followed for 8 weeks. Sacubitril/valsartan compared to placebo reduced the BP by $11.5 / 4.7 \mathrm{mmHg}$ with the $100 \mathrm{mg}$ dose, by $11.0 / 6.5 \mathrm{mmHg}$ with the $200 \mathrm{mg}$ dose and by $12.5 / 7.8 \mathrm{mmHg}$ with the 400 $\mathrm{mg}$ dose. In another major study, Williams et al. (7), tested the effects of sacubitril/valsartan versus olmesartan on BP lowering and arterial stiffness in 454 patients mean age 67.7 years in the Prospective Comparison of an AngiotensinReceptor Neprilysin Inhibitor With an AngiotensinReceptor Blocker Measuring Arterial Stiffness in the Elderly (PARAMETER) study. After an initial randomization to sacubitril/valsartan $200 \mathrm{mg} /$ day or olmesartan $20 \mathrm{mg} /$ day, the doses were increased to sacubitril/valsartan $400 \mathrm{mg} / \mathrm{day}$ or olmesartan $40 \mathrm{mg} /$ day and were followed for 52 weeks. Depending on BP response, amlodipine 2.5 to $5.0 \mathrm{mg} /$ day plus hydrochlorothiazide 6.25 to $25.0 \mathrm{mg}$ /day were added. The sacubitril/valsartan $400 \mathrm{mg}$ regimen reduced the BP by 17.7/8.7 $\mathrm{mmHg}$ compared to olmesartan $40 \mathrm{mg}$ /day regimen of 16.1/8.1 mmHg. The effects on BP and pulse pressure (PP) were similar between the two drug regimens. The drugs were well tolerated without major clinical or metabolic side effects. In another study Cheung et al. (15), compared the effects of sacubitril/valsartan $200 \mathrm{mg} /$ day against olmesartan $20 \mathrm{mg} /$ day in 376 hypertensive patients mean age 57.6 years. After an 8-week follow-up, sacubitril/valsartan decreased the clinic BP by $14.2 / 7.5 \mathrm{mmHg}$ compared to olmesartan of 10.0/4.5 $\mathrm{mmHg}$ and the 24-hour ABP by $4.3 / 2.3 \mathrm{mmHg}$ compared to olmesartan of 1.1/0.4 $\mathrm{mmHg}$. Both types of BP measurements were significantly lower with sacubitril/valsartan compared to olmesartan. Also the $\mathrm{PP}$ was reduced greater with sacubitril/valsartan compared to olmesartan. Sacubitril/valsartan either alone or in combination with other antihypertensive drugs has been tested in other smaller studies $(6,13,14)$ and their results are summarized in Table 1.

\section{Effects of sacubitril/valsartan in patients with heart failure}

Sacubitril/valsartan has been shown to have beneficial effects in patients with HFrEF as well as in those with HF preserved $\mathrm{EF}(\mathrm{HFpEF})$ and at present is the drug indicated only for the extended treatment of HFrEF. McMurray et al. (1) investigated the effects of sacubitril/valsartan monotherapy in comparison with enalapril monotherapy in the PARADIGM in Heart failure (PARADIGM-HF) study on 8,442 patients mean age $63 \pm 11.5$ years with HFrEF (EF $\leq 40 \%)$. The patients were randomized to 
either sacubitril/valsartan $200 \mathrm{mg}$ twice daily or enalapril $10 \mathrm{mg}$ twice daily and were followed for a median of 27 months (premature termination). At study end, the primary outcome (a composite of death from cardiovascular causes or hospitalization for HF) occurred in $21.8 \%$ in patients treated with sacubitril/valsartan and in $26.5 \%$ in those treated with enalapril, hazard ratio (HR) 0.80 (95\% CI, $0.73-0.87, \mathrm{P}<0.001)$. A total of $13.3 \%$ and $16.5 \%$ of patients treated with sacubitril/valsartan or enalapril, respectively died from cardiovascular causes, HR 0.80 (95\% CI, 0.71-0.89, $\mathrm{P}<0.001$ ). Instances of hypotension, and increases in serum creatinine and potassium levels were small and similar between the two drug regimens. Cough or angioedema occurred in $0.2 \%$ of patients treated with sacubitril/valsartan versus $0.1 \%$ in those treated with enalapril $(\mathrm{P}=0.19)$. In addition a meta-analysis by Solomon et al. (16), of the PARADIGM-HF, IMPRESS, and OVERTURE trials showed that the composite outcome of death or HF hospitalization was reduced numerically in patients receiving a combined NEP/ACE inhibition compared with ACE inhibition alone with a pooled HR of 0.86 (95\% CI, 0.76-0.97, P=0.013). For the all-cause mortality the pooled HR was 0.88 (95\% CI, $0.80-0.98, \mathrm{P}=0.021)$ for the combined regimen compared to monotherapy. Combined NEP/ACE inhibition resulted in more instances of hypotension, but lesser instances of renal dysfunction and hyperkalemia than ACE inhibition alone. Also, a recent secondary analysis of the results of the PARADIGM-HF trial by Chandra et al. (17), sacubitril/valsartan improved nearly all the physical and social activities of the Kansas City Cardiomyopathy Questionnaire compared to enalapril with the largest responses in household chores and sexual relationships. In addition to patients with HFrEF, the effects of sacubitril/ valsartan were tested on NT-pro BNP levels in patients with HFpEF in the Prospective Comparison of ARNI with ARB on Management of HF with Preserved Ejection Fraction (PARAMOUNT) trial (2). This trial was a phase II, randomized, parallel-group, double-blind multicenter study of 301 patients mean age of 71 years, with NYHA class II-III wit EF $\geq 45 \%$ and NT-proBNP $>400 \mathrm{pg} / \mathrm{dL}$. The patients were randomized to either sacubitril/valsartan $200 \mathrm{mg}$ or valsartan $160 \mathrm{mg}$ twice daily in addition to background therapy except ACE inhibitors or ARBs and followed for 12 weeks with an extension to 36 weeks. At 12 weeks, the NP-proBNP levels were decreased with sacubitril/valsartan from a baseline of 783 to $605 \mathrm{pg} / \mathrm{dL}$ and with valsartan from a baseline of 862 to $835 \mathrm{pg} / \mathrm{dL}$ with a ratio sacubitril/valsartan/valsartan 0.77 (95\% CI, 0.64-0.92, $\mathrm{P}=0.005)$. However, at week 36 there was no difference in the decrease of NP-proBNP between the two treatment regimens. Of interest, was the greater decrease of NT-proBNP with valsartan in diabetic patients $(\mathrm{P}=0.02)$. A similar long-term phase III study, a Prospective Comparison of ARNI with ARB Global Outcomes in HF With Preserved Ejection Fraction (PARAGON-HF) trial in patients with $\mathrm{HFpEF}$ ( $\mathrm{EF} \geq 45 \%$ ) is in progress. These patients are being treated with sacubitril/valsartan $100 \mathrm{mg}$ twice daily compared to valsartan $80 \mathrm{mg}$ twice daily. The primary outcome is the composite of cardiovascular death and HF hospitalizations

\section{Other important actions of sacubitril/valsartan}

Sacubitril/valsartan is a dual molecule consisting of the NEP inhibitor sacubitril and the Ang II, AT1 receptor blocker valsartan in 1:1 molar ratio (18). The NEP is known to catabolize several peptide hormones such as adrenomedullin, angiotensin II, bradykinin, endothelin, glucagon like peptide-1 (GLP-1), NPs, neurotensin, oxytocin, substance $\mathrm{P}$, and most importantly amyloid $\beta$ peptide. The main actions of these peptide hormones are summarized in Table 2. Consequently, the net effect of NEP inhibition will be a balance between the decrease in BP by the blockade of degradation of adrenomedullin, bradykinin and NPs through vasodilation and natriuresis or increase in BP through the blockade of degradation of Ang II and endothelin-1 as has been demonstrated in healthy men given a NEP inhibitor (19). However, in the case of sacubitril/valsartan the effects of Ang II will be inhibited through the blockade of AT1 receptor with valsartan. The blockade of degradation of bradykinin and substance $\mathrm{P}$ could cause cough and angioedema, but these adverse effects are not very common with sacubitril/valsartan. The most worrisome effect of long-term treatment with sacubitril/valsartan is the inhibition of degradation of amyloid $\beta(\mathrm{A} \beta)$ peptide and the possible increased incidence of Alzheimer's disease, since NEP has been touted as a drug to prevent Alzheimer's disease (20). Another possible mechanism for the beneficial effects of sacubitril/valsartan was investigated by Engeli et al. (12) in their recent study. They investigated the fat tissue lipolysis and fat mobilization, with the combination of sacubitril/valsartan and exercise with an elaborate microdialysis technique and glycerol tracer kinetics in 98 obese hypertensive subjects, waist circumference $(W C \geq 102 \mathrm{~cm}$ in men and $\geq 88 \mathrm{~cm}$ in 
Table 2 Main actions of peptide hormones catabolized by neprilysin

\begin{tabular}{ll}
\hline Name & Action \\
\hline Adrenomedullin & Circulatory peptide hormone causing systemic vasodilation and new angiogenesis \\
Angiotensin II & Circulatory peptide hormone causing systemic vasoconstriction and cardiovascular remodeling \\
Bradykinin & Circulatory peptide hormone causing systemic vasodilation and cough stimulation \\
Endothelin-1 & Circulatory peptide hormone causing systemic vasoconstriction \\
Glucagon & Peptide hormone released from the $\alpha$-cells of the pancreas catalyzing glycogen and increasing blood glucose levels \\
Natriuretic & Circulatory peptide hormones causing systemic vasodilation and increasing diuresis and natriuresis \\
peptides & Circulatory neuropeptide hormone regulating the release of luteinizing and prolactin hormone release and causing \\
Neurotensin & systemic vasodilation through its interaction with the dopaminergic system \\
Oxytocin & Circulatory peptide hormone released from the paraventricular nucleus of the brain and facilitating child birth \\
Substance $P$ & Circulatory neuropeptide hormone acting as neurotransmitter and neuromodulator and stimulating cough production \\
Amyloid $\beta$ & Increased brain deposition of amyloid $\beta$ has been implicated as a cause of Alzheimer's disease
\end{tabular}

women and $130 \leq \mathrm{SBP}<180 \mathrm{mmHg}$ ). The patients were randomized to sacubitril/valsartan $400 \mathrm{mg} /$ day or to a neutral comparator amlodipine $10 \mathrm{mg}$ /day and followed for 8 weeks. The rationale for this study was based on the knowledge that NPs have been shown to cause weight loss, increase insulin sensitivity and decrease the incidence of T2DM, and of a potent lipolytic effect in human-isolated adipocytes (21). Therefore, sacubitril by blocking the NEP catabolism of NPs it was expected to increase the abdominal and whole body lipolysis in conjunction with exercise. However, this study demonstrated that sacubitril/valsartan increased the abdominal, but not the whole body lipolysis compared to amlodipine. This is strange and makes no sense, but was not explained by the investigators. However, in a previous similar study by the same investigators, whole body lipolysis was not increased with sacubitril/valsartan in contrast to increased abdominal lipolysis and the authors suggested that this difference could be due to a balanced effect by decreased lipolysis in other parts of the body, although this is not known at present (22). Therefore, according to these studies, fat tissue lipolysis does not appear to explain the beneficial effects of sacubitril/valsartan in obese patients with hypertension $(12,22)$, although other investigators have demonstrated increased lipolysis and weight loss with sacubitril/valsartan $(21,23)$. Other investigators have reported low levels of NPs in patients with diabetes and obesity (11). This controversy regarding the lipolytic or not effects of sacubitril/valsartan needs to be confirmed by future studies.

\section{Discussion}

From the available data, it appears that sacubitril/valsartan is a cardiovascular drug with pluripotential actions. It possesses significant beneficial effects in patients with hypertension, HF, and T2DM through vasodilation, natriuresis, weight loss and increase in insulin sensitivity and it is fairly safe and well tolerated (8-10). In addition, its lowering effects on blood levels of NT-proBNP are also significant since high levels of NT-proBNP predict the future onset of cardiovascular events (24). Its effects on different substrates appear to balance each other and not to present any adverse effects with the exception on its effects on the prevention of degradation of $A \beta$ peptide and possible increase in the incidence of Alzheimer's disease after prolonged treatment $(20,25)$. The worry is not so much for patients with $\mathrm{HF}$, because of their short survival, but it could be significant for patients with hypertension, because of their long survival with effective antihypertensive treatment. The other possible beneficial effects of sacubitril/ valsartan on weight loss by whole body lipolysis were not demonstrated by Engeli et al. (12), although have been demonstrated by other investigators $(11,23)$. The difference between the increased abdominal lipolysis with sacubitril/ valsartan and not the whole body lipolysis is not discussed by the authors, although it could be due to a balanced effect of decreased lipolysis elsewhere in the body, although increased lipolysis with NPs has been suggested by other investigators (21). The significant increase of noradrenaline 
levels with exercise in both treatment groups shown in this study, is worrisome, although it did not interfere with the lowering of BP. This effect could be possibly due to sacubitril/valsartan inability to suppress the elevation of noradrenaline levels with exercise.

In conclusion, sacubitril/valsartan is a pluripotential cardiovascular drug with beneficial effects in patients with hypertension and $\mathrm{HF}$, without significant adverse effects. Its long-term effects on the incidence of Alzheimer's disease were worrisome, but awaits confirmation by future studies.

\section{Acknowledgements}

None.

\section{Footnote}

Conflicts of Interest: The authors have no conflicts of interest to declare.

\section{References}

1. McMurray JJ, Packer M, Desai AS, et al. Angiotensinneprilysin inhibition versus enalapril in heart failure. $\mathrm{N}$ Engl J Med 2014;371:993-1004.

2. Solomon SD, Zile M, Pieske B, et al. The angiotensin receptor neprilysin inhibitor LCZ696 in heart failure with preserved ejection fraction: a phase 2 double-blind, randomised controlled trial. Lancet 2012;380:1387-95.

3. Ruilope LM, Dukat A, Böhm M, et al. Blood pressure reduction with LCZ-696, a novel dual-acting inhibitor of the angiotensin II receptor and neprilysin: a randomized, double blind, placebo-controlled active comparator study. Lancet 2010;375:1255-66.

4. Chrysant SG. Pharmacokinetic, pharmacodynamic, and antihypertensive effects of the neprilysin inhibitor LCZ-696: sacubitril/valsartan. J Am Soc Hypertens 2017;11:461-8.

5. Kario K, Sun N, Chiang FT, et al. Efficacy and safety of LCZ696, a first-in-class angiotensin receptor neprilysin inhibitor, in Asian patients with hypertension. A randomized, double-blind, placebo-controlled study. Hypertension 2014;63:698-705.

6. Kario K, Tamaki Y, Okino N, et al. LCZ696, a first-inclass angiotensin receptor-neprilysin inhibitor: The first clinical experience in patients with severe hypertension. J Clin Hypertens (Greenwich) 2016;18:308-14.

7. Williams B, Cockroft JR, Kario K, et al. Effects of sacubitril/valsartan versus olmesartan on central hemodynamics in the elderly with systolic hypertension. The PARAMETER study. Hypertension 2017;69:411-20.

8. Rubattu S, Sciarretta S, Valenti V, et al. Natriuretic peptides: an update on bioactivity, potential therapeutic use, and implications in cardiovascular diseases. Am J Hypertens 2008;21:733-41.

9. Mangiafico S, Costello-Boerringer LC, Anderson IA, et al. Neutral endopeptidase inhibition and the natriuretic peptide system: an evolving strategy in cardiovascular therapeutics. Eur Heart J 2013;34:886-93.

10. Wang TJ, Larson MG, Keyes MJ, et al. Association of plasma natriuretic peptide levels with metabolic factors in ambulatory individuals. Circulation 2007;115:1345-53.

11. Magnusson M, Jujic A, Hedblad B, et al. Low plasma level of atrial natriuretic peptide predicts development of diabetes: the prospective Malmo Diet and Cancer Study. J Clin Endocrinol Metab 2012;97:638-45.

12. Engeli S, Stinkens R, Heise T, et al. Effects of sacubitril/ valsartan on exercise-induced lipid metabolism in patients with obesity and hypertension. Hypertension 2018;71:70-7.

13. Ito $S$, Satoh M, Tamaki Y, et al. Safety and efficacy of LCZ696, first-in-class angiotensin receptor neprilysin inhibitor, in Japanese patients with hypertension and renal dysfunction. Hypertens Res 2015;38:269-75.

14. Wang JG, Yukisada K, Sibuto A Jr, et al. Efficacy and safety of sacubitril/valsartan (LCZ696) add-on to amlodipine in Asian patients with systolic hypertension uncontrolled with amlodipine monotherapy. J Hypertens 2017;35:877-85.

15. Cheung DG, Aizenberg D, Gorbunov V, et al. Efficacy and safety of sacubitril/valsartan in patients with essential hypertension uncontrolled by olmesartan: a randomized, double-blind, 8 week study. J Clin Hypertens (Greenwich) 2018;20:150-8.

16. Solomon SD, Claggett B, McMurray JJ, et al. Combined neprilysin and renin-angiotensin system inhibition in heart failure with reduced ejection fraction: a meta-analysis. Eur J Heart Fail 2016;18:1238-43.

17. Chandra A, Lewis EF, Claggett BL, et al. Effects of sacubitril/valsartan on physical and social activity limitations in patients with heart failure: A secondary analysis of the PARADIGM-HF trial. JAMA Cardiol 2018. [Epub ahead of print].

18. Gu J, Noe A, Chandra P, et al. Pharmacokinetics and phatmacodynamics of LCZ696, a novel dual-acting angiotensin receptor-neprilysin inhibitor (ARNI). J Clin Pharmacol 2010;50:401-14. 
19. Ando S, Rahman MA, Butler GC, et al. Comparison of candoxatril and atrial natriuretic factor in healthy men. Effects of hemodynamics, sympathetic activity, heart rate variability, and endothelin. Hypertension 1995;26:1160-6.

20. Ashby EL, Miners JS, Kehoe PG, et al. Effects of hypertension and antihypertensive treatment on amyloid $\beta(A \beta)$ plaque load and $A \beta$-degrading enzymes in frontal cortex. J Alzheimers Dis 2016;50:1191-203.

21. Sengenès C, Berlan M, De Gliseczinski I, et al. Natriuretic peptides: a new lipolytic pathway in human adipocytes. FASEB J 2000;14:1345-51.

22. Jordan J, Stinkens R, Jax T, et al. Improved insulin sensitivity with angiotensin receptor neprilysin inhibition

Cite this article as: Chrysant SG, Chrysant GS. Sacubitril/ valsartan: a cardiovascular drug with pluripotential actions. Cardiovasc Diagn Ther 2018;8(4):543-548. doi: 10.21037/ cdt.2018.05.10 in individuals with obesity and hypertension. Clin Pharmacol Ther 2017;101:254-63.

23. Moro C. Natriuretic peptides and fat metabolism. CCurr Opin Clin Nutr Metab Care 2013;16:645-9.

24. Chrysant SG. The clinical significance of $\mathrm{N}$-terminal Pro-brain Natriuretic peptide in detecting the residual cardiovascular risk in hypertension and other clinical conditions and in predicting future cardiovascular events. J Clin Hypertens (Greenwich) 2016;18:718-20.

25. Langenickel TH, Tsubouchi C, Ayalosomayajula S, et al. The effect of LCZ696 (sacubitril/valsartan) on amyloidbeta concentrations in cerebrospinal fluid in healthy subjects. Br J Clin Pharmacol 2016;81:878-90. 